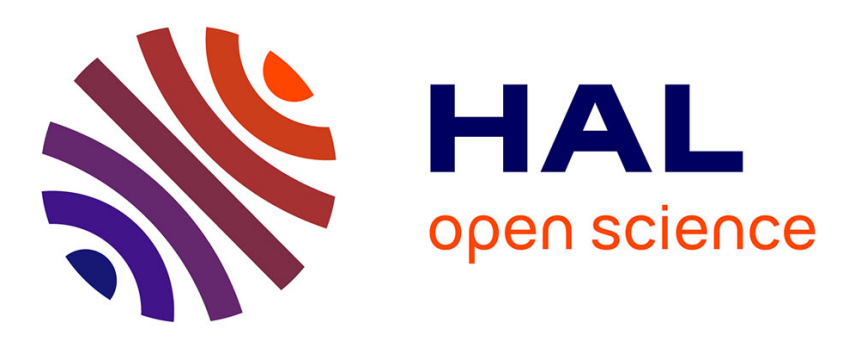

\title{
Study and Evaluation of Laser-based Perception and Light Communication for a Platoon of Autonomous Vehicles
}

Mohammad Abualhoul, Pierre Merdrignac, Oyunchimeg Shagdar, Fawzi

Nashashibi

\section{To cite this version:}

Mohammad Abualhoul, Pierre Merdrignac, Oyunchimeg Shagdar, Fawzi Nashashibi. Study and Evaluation of Laser-based Perception and Light Communication for a Platoon of Autonomous Vehicles. 2016 IEEE 19th Intelligent Transportation Systems Conference (ITSC 2016), Nov 2016, Rio de Janeiro, Brazil. hal-01366147

\section{HAL Id: hal-01366147 \\ https://hal.inria.fr/hal-01366147}

Submitted on 14 Sep 2016

HAL is a multi-disciplinary open access archive for the deposit and dissemination of scientific research documents, whether they are published or not. The documents may come from teaching and research institutions in France or abroad, or from public or private research centers.
L'archive ouverte pluridisciplinaire HAL, est destinée au dépôt et à la diffusion de documents scientifiques de niveau recherche, publiés ou non, émanant des établissements d'enseignement et de recherche français ou étrangers, des laboratoires publics ou privés. 


\title{
Study and Evaluation of Laser-based Perception and Light Communication for a Platoon of Autonomous Vehicles
}

\author{
Mohammad Y. Abualhoul ${ }^{\dagger}$, Pierre Merdrignac ${ }^{\dagger *}$, Oyunchimeg Shagdar ${ }^{\dagger *}$ and Fawzi Nashashibi ${ }^{\dagger}$ \\ RITS Team - INRIA Paris, 2 Rue Simone IFF, 75012 Paris, France ${ }^{\dagger}$ \\ VEDECOM Institute, 77, rue des Chantiers, 78000 Versailles, France* \\ \{mohammad.abu_alhoul, pierre.merdrignac, oyunchimeg.shagdar, fawzi.nashashibi\}@inria.fr
}

\begin{abstract}
Visible Light Communication (VLC) is a new emerging technology that is being proposed as a reliable and supportive choice for short range communications in ITS. On the same context, Laser Range Finders (LRF) sensors are used for the vehicular environment perception. Compared to VLC, LRF can provide more coverage range and extended viewing angle. To take the full advantages of both technologies features, this paper studies and demonstrate the proposal of using VLC for information exchange among the platoon members and LRF for inter-vehicle distance estimation. A hand-over algorithm is proposed to manage the switching process for any failure occurrence by assessing LRF and VLC performance using three different metrics: LRF confidence value, vehicles angular orientation, and the VLC link latency. The evaluation of the proposed system is verified using VLC prototype and ProSiVIC Simulator driving platoon of two autonomous vehicles over different curvature scenarios. Our results show that the proposed combination are extending the VLC limitations and satisfying the platooning requirement. However, in the very sharp curvature, LRF was capable of driving the platoon except for the $90^{\circ}$ curve scenario, the system experienced non-stable behaviour due to the LRF area of interest limitation.
\end{abstract}

Keywords: VLC, Laser sensors, Platoon, ITS, Road safety applications.

\section{INTRODUCTION}

Intelligent transport systems (ITS) can provide more safe and secure traffic by properly utilizing the information technology. Such information can be acquired using the vehicular embedded sensors, which provides the necessary data to perceive the vehicle's surrounding environment. Furthermore, inter-vehicle communication and the Cooperative ITS (CITS) mode can also play a significant role in improving road traffic safety and comfort [1]. In such context, communication between vehicles $(\mathrm{V} 2 \mathrm{~V})$, vehicles sensors range and viewing capability are one of the critical issues for C-ITS.

As proposed solutions for traffic congestion, automobile manufacturers have developed advanced driver assistant systems such as Adaptive Cruise Control (ACC), which intend to improve driving comfort. ACC uses sensors such as cameras, laser or radars to estimate the relative distance and relative velocity of two nearby vehicles [2]. Vehicles with ACC are capable of changing acceleration automatically to adapt desired distance and sustain a safe distance from the preceding vehicle. Moreover, the Cooperative Adaptive Cruise Control (CACC) systems are introduced as an improvement to the ACC by exchanging information between vehicles using radio communication. Beside improve driving comfort, the main objective of CACC is to improve the traffic flow and save energy in congested scenarios. Compared with ACC, CACC can keep relatively smaller headway time, which can enhance traffic flow dramatically, and improves the system stability by providing the assessment of approaching future situations and also result in better decision-making and prediction.

Due to the accelerated increase of the wireless-based communication devices numbers, well-known wireless communication technologies such as the standardized IEEE 802.11p technology for ITS [3] are pushed toward an insatiable demand for wireless networks data access, with a remarkable increase in both latency and channel congestion levels. This instability introduced more usage constraints for ITS applications [4], especially for the hard-safety use, such as platooning. Different communication technologies using another communication medium such as Visible light communication (VLC) has become an outstanding intriguing supportive technology lately. Realizing both low-cost implementation and dual functionality, VLC has also the advantage of using the vehicular existed infrastructure, (e.g. rear and front lamps of the vehicle) as transmitters.

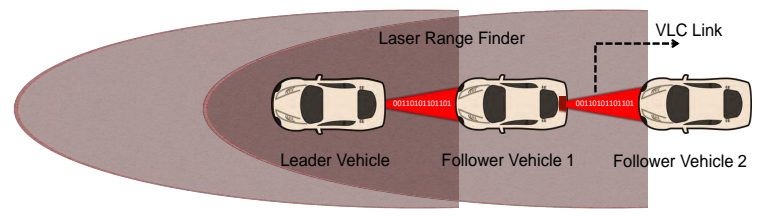

Fig. 1. VLC and LRF Systems for platooning application.

The main benefits of using VLC in such configuration is to activate the CACC mode between platoon members without introducing any load to the radio communication channel. Although that VLC is sensitive to the vehicular orientation, holding a reliable backup perception sensing able to drive the platoon in ACC mode with a sufficient hand-over can overcome this limitation. Fig. 11 illustrates the field of view (FOV) of embedded sensors (laser) and VLC communication range. Such configuration intends to utilize both technologies to ensure platoon application safety requirements.

Laser Range Finders (LRF) can have wide FOV with a sensing range up to $100 \mathrm{~m}$, which is sufficient to track the preceding vehicle in a platoon. On the other hand, VLC operates in relatively narrower FOV, and has communication 
range up to $15 \mathrm{~m}$.

In this paper, we study and evaluate the advantage of using VLC and LRF for platooning. By combining both solutions, the vehicular status and GPS positions are exchanged among the platoon members using the vehicular rear lighting system. Further, the LRF is operating in redundant mode as a backup for the VLC blackout. We concentrate on observing the overall platoon behaviour for different trajectory scenarios, intended to drive the VLC link to its FOV limits by introducing communication failure and evaluate the limits of our system. In this particular scenes, our study highlights the switching modes between the presence of VLC and disconnections cases, in other words, the availability of CACC and $\mathrm{ACC}$, recognising the platoon minimum safety requirements. In this paper, we make the following contributions:

- Suggesting a new architecture intend to improve the platoon performance by utilizing both LRF and VLC.

- Studying the limitations and performance of both technologies for various car-following scenarios (straightline, lane-change and curve).

- Proposing hand-over algorithm to check the platoon safety requirements and the availability of ACC and CACC modes.

- Evaluating the system performance and the hand-over mechanism between cooperative and LRF-only platooning modes.

The remainder of this paper is organized as follows. Section II discusses the previous literature and related work. The proposed system to drive platoon using VLC and LRF including the used models is discussed in Section III. Section IV details the hand-over algorithm proposal. After describing the system structure in Section V, we report the system performance and our findings in Section VI. Conclusion and planned future work are given in Section VII.

\section{RELATED WORKS}

Over the last thirty year, platooning concept has been proposed to improve the traffic throughput and increase roads capacity. PATH project in California [5] and PRAXITELE project in France [6] were one of the first pioneering projects demonstrating the platoon. Later proceeding, Auto21 CDS [7] project was dedicated to advance the smooth merging/leaving for a platooning-enabled system. Also, the SARTRE project [8] demonstrated the fully autonomous platoon excluding the manually-driven leader, where all following vehicles are free to merge and/or leave the platoon in a fully autonomous mode.

By mapping the environment using infrared beams [9], LRF can detect and precisely localize obstacle with up to $360^{\circ}$ FOV [10], [11]. Thus, they can be mounted on every platoon member to estimate the inter-vehicle distance. However, using such sensors might introduce some imprecision when estimating the preceding vehicle velocity, especially when vehicles follow sharp road curvatures. The vehicular status exchange between platoon members (V2V) communication was the straight-forward proposal to overcome these types of limitations [12]. In this paper, we introduce an area of interest using the vehicle motion model in order to identify more exactly the vehicle in front and to estimate its position.

Authors in [12] represented vehicular platoon model based on constant inter-vehicle spacing. In this model, the platoon members receive broadcasted event-triggered messages over Dedicated Short Range Communications (DSRC), while a periodic vehicle control-based messages between platoon members utilize an infra-red (IR) spectrum to communicate in unicast mode. Although that IR is a popular medium for optical LOS communications, still compared to VLC, the use of IR spectrum considered costly with strict safety requirements. We have also contributed in theoretically studying the VLC channel characteristics and the technology feasibility for ITS applications [13],[14].

Experimental studies on VLC for outdoor applications are conducted as presented in [15]. The studies show that VLC can satisfy the stringent reachability requirements for Inter-Vehicle Communication (IVC) in dense vehicle traffic conditions. The authors also presented a prototype of vehicular system proposing a VLC down link between road infrastructures and vehicles, aiming to enhance traffic security.

The main requirement of platoon controller concerns the longitudinal control [16], [17]. ACC with a constant time headway policy has been a common solution to maintain a safe distance between platoon members [17]. Then, it has been shown that one of the main advantages of using both communication and sensors in a Cooperative ACC (CACC) mode over only sensors in an ACC mode, is the decrease the inter-vehicle time headway from $1.1 \mathrm{~s}$ to $0.6 \mathrm{~s}$ while satisfying the safety requirement and maintaining the string stability within the platoon [17]. In our paper, we study and evaluate the conditions for relying on either ACC or CACC modes in a platoon using VLC communications and LRF. In addition, we propose a hand-over method to ensure the safety with ACC mode when IVC is not available.

\section{Platoon DRIVING USING VLC AND LRF}

The proposed system differs from the earlier discussed related research in the way that we demonstrate the deployment of two different technologies ensuring stable platoon behavior. Benefiting from the adaptability of the laser sensors for detection and tracking in the ACC mode, and using the vehicle commercial lighting system to establish a low-cost communication link between platoon members, we can rely on the CACC mode as long as the VLC is active.

This approach allows us to overcome the introduced errors when using laser sensors to estimate speed and inter-vehicle distance, at the same time, keeping a backup when the VLC blackout occurs due to the FOV limitations. In our study, we propose to use VLC to exchange vehicular status (e.g., speed, orientation, acceleration) and relative locations within platoon members, redundantly with the laser sensors. In this way, the proposed system can rely on two independent information sources for taking the best decision for platooning. To our best knowledge, there is no published 
research concerning the combination of both technologies for platooning.

Figure 2 shows our proposed platooning system, where each platoon member is equipped with LRF to measure the distance with its preceding vehicle and ensure a safe platoon by means of ACC longitudinal control. Vehicles are also equipped with a VLC transmitters and receivers such that the platoon members can rely on a CACC longitudinal control. LRF has FOV of $180^{\circ}$ with a sensing range up to $100 \mathrm{~m}$, where VLC operates in relatively narrower FOV, and has a communication range of $30 \mathrm{~m}$, suitable to establish InterVehicle Communication (IVC). The FOV for LRF and VLC in our system depicted in Fig. 2 and defined as the following:

- Laser-Range-Finder (LRF) is mounted on the follower vehicle, allowing the follower vehicle to detect reflected beams from the preceding one. Thus, the LRF-FOV is defined to be from the follower vehicle end toward the preceding one.

- VLC uses the leader vehicle rear-light system as a communication transmission unit, where the receiver is mounted on the follower vehicle. Hence, VLC-FOV is defined by the Light Emitting Diode (LED)'s Lambertian characteristics from the preceding vehicle end toward the follower vehicle.

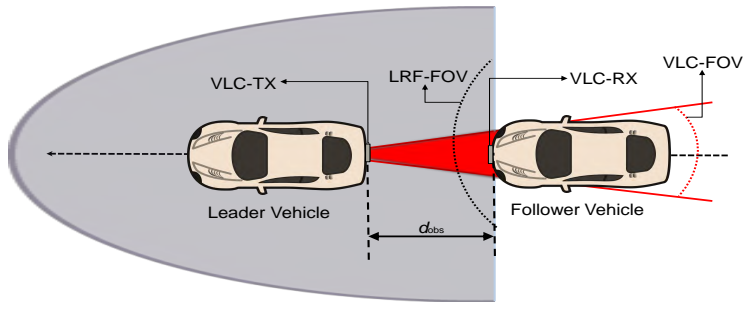

Fig. 2. Two autonomous vehicles platoon using LRF and VLC.

Among 75 ITS services recognized by the department of US transportation, eight services were classified as hard safety requirements [18]. Regardless the technology-in-use, the highest priority safety services such as crash avoidance and lane change warning target $90-100 \%$ of data update Packet Delivery Ratio (PDR), and maximum end-to-end latency of 10 to $100 \mathrm{~ms}$. In our study, we investigate the VLC and LRF models and the system implementation by considering a platoon application with hard safety requirements.

\section{A. Laser Based Detection Model}

By using laser sensors, obstacles are detected, classified and their position regarding the sensor is estimated [19]. In a platoon formation, as the preceding vehicle is close enough, we can rely on laser based detection algorithm to estimate its distance $d_{o b s}$ with the platoon member [11]. Such algorithm also provides a confidence value $C$ on the detected vehicle that can reach $100 \%$ after a certain number of successful observations.

However, many obstacles fall within LRF-FOV and the preceding vehicle has to be correctly identified. Since this particular obstacle is supposed to be in the near future trajectory of the platoon member, it is searched in an area of interest of width $W_{\text {Area }}$ around the predicted vehicle trajectory $\left(x_{p}(t), y_{p}(t)\right)$ as illustrated in Fig. 3 .

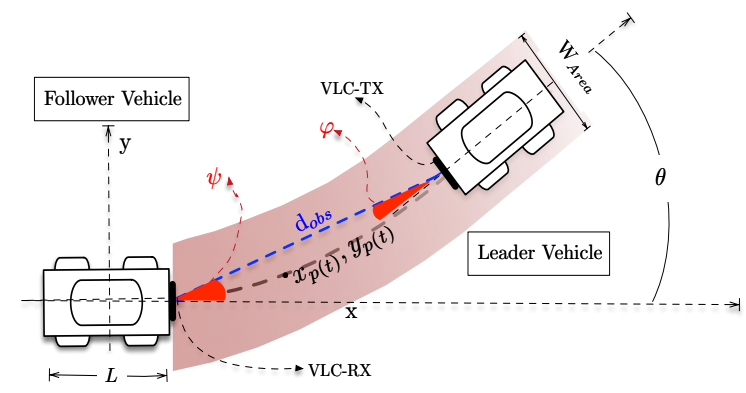

Fig. 3. Laser based preceding vehicle detection and VLC for two platoon members. $L$ represent the vehicle length and $W_{\text {Area }}$ is width of the laser detection area of interest. $\psi$ and $\varphi$ are VLC incidence and irradiance angles.

In our implementation, a bicycle model is used to predict the vehicle trajectory points from its current velocity $v_{p}$ and steering angle $\varepsilon_{p}$, using the following equation:

$$
\begin{aligned}
& x_{p}(t)=\frac{L}{\varepsilon_{p}} \sin \left(\frac{L \times v_{p}}{\varepsilon_{p}} t\right) \\
& y_{p}(t)=\frac{L}{\varepsilon_{p}}\left(1-\cos \left(\frac{L \times v_{p}}{\varepsilon_{p}} t\right)\right),
\end{aligned}
$$

where $L$ represent the vehicle length.

\section{B. Visible Light Communication Model}

VLC channel characteristics can be defined mainly by the LED lambertian emission pattern and both transmitter and receiver physical characteristics [20], where the path-loss can be presented as:

$$
P_{\text {loss }}=\left\{\begin{array}{cc}
\frac{(m+1) A_{p h}}{2 \pi d^{n}} \cos ^{m}(\varphi) \cos (\psi), & 0<\psi, \varphi<\theta_{c} \\
0 & \text { elsewhere }
\end{array} .\right.
$$

For VLC path-loss theoretical model, together with an experimental results obtained from the implemented prototype [21], the path-loss exponent $n$ is equal to 2, where $d$ is the separation distance between transmitter and receiver, $\theta_{c}$ is the maximum incidence and irradiance angles $\max (\psi, \varphi)$, which is also our system VLC-FOV limits as depicted in Fig. 3. $A_{p h}$ is the physical area of the PD and $m=-\frac{\ln 2}{\ln (\cos \hat{\phi})}$ is the lambertian emission order, which is a key parameter specifying the directivity of the transmitter. The LED halfpower angle $\hat{\phi}$ has a remarkable influence on the coverage range and pattern shape of the lambertian light source. By choosing narrower $\hat{\phi}$ or mounting the LED with an optical lens, we can directly change the VLC-FOV and increase the communication range.

\section{HAND-OVER BETWEEN VLC AND LRF}

In our system, the cooperative platooning mode can be possible only when both LRF and VLC are available and satisfy the hard safety requirements. Initially, the minimum configuration considers LRF for estimating the inter-vehicle 
distance in order to rely on an ACC mode. The ideal configuration is when VLC is meeting the same requirements and starts to exchange the preceding vehicle velocity, orientation and position. For this configuration, the system can rely on a CACC mode. Thus, a hand-over is required between these two modes based on the availability of LRF and VLC. Our proposed hand-over is influenced by three main metrics that characterise and track the limitations of LRF and VLC as the following:

1) The laser based detection confidence $C$ is the main LRF metric, this value is found after certain number of detections. Once the leader vehicle is correctly detected with $C \geq 90 \%$, the leader vehicle identifier $L_{I D}$ is passed and ACC mode is active.

2) The maximum of $\varphi, \psi$ and $\theta$, which represent the VLC transmitter and receiver angular difference with respect to the relative orientation and the lane change. If any of the tracked angles exceeds $\zeta=80 \%$ of the critical FOV limit $\left(\theta_{c}\right)$, the decision then handing the LRF output before reaching the VLC disconnection.

3) End-to-end delay $\tau$, this condition continuously checks the safety requirement, where the delay must remain under the $100 \mathrm{~ms}$ limit. Also, the exceeded delay can indicate VLC disconnection due to an obstacle presence even though transmitter and receiver might be perfectly aligned.

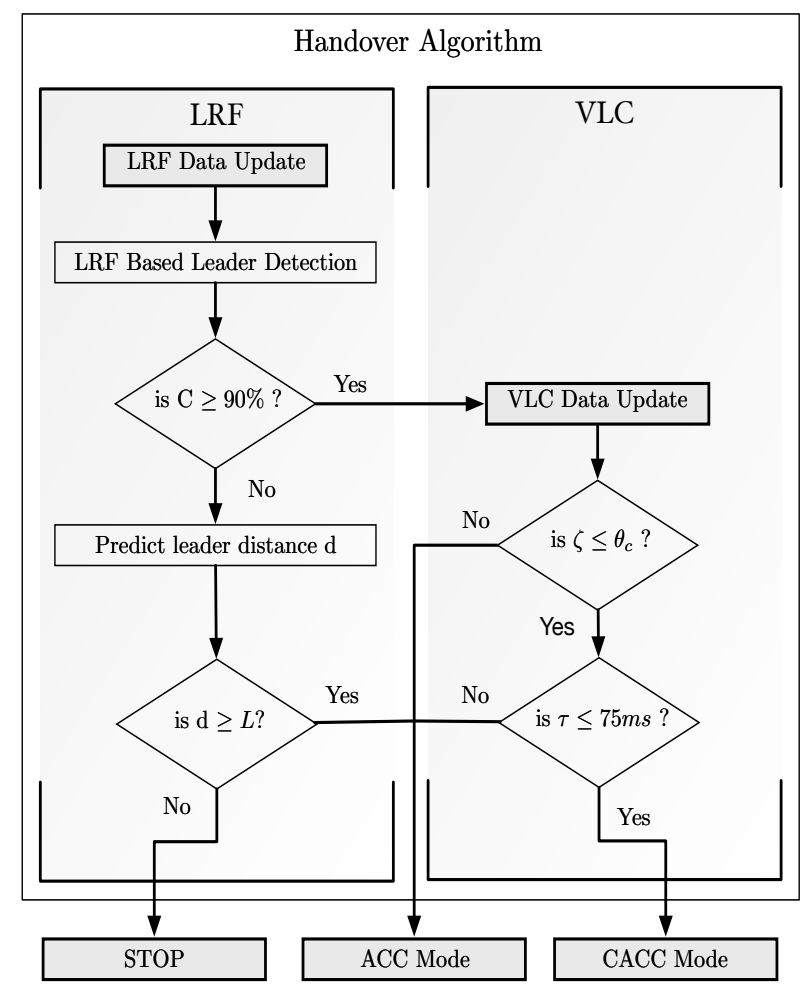

Fig. 4. Flow chart of hand-over between VLC and LRF.

Our proposed hand-over algorithm has two main input and three output as illustrated in Fig. 4. Both VLC and LRF provides data update simultaneously where the algorithm output is the availability of either ACC, CACC or STOP modes that satisfy the platoon safety requirements.

As LRF is the minimum and initial configuration for our system, the algorithm is activated with laser data update. The preceding vehicle has to be detected and its identifier $L_{I D}$ is tracked over time while it remains in the area of interest. At each time instant, the LRF confidence of detecting the leader vehicle $C$ is compared to a given threshold to establish if a cooperative platoon can be considered. When $C \leq 90 \%$, the track of the leader vehicle is considered lost, and the inter-vehicle distance $d_{t}$ is predicted based on the previous estimated inter-vehicle distance $d_{t-1}$ using eq. (3), where the sensor update period $T_{0}$ and current vehicle velocity $v_{p}$.

$$
d_{t}=d_{t-1}-\left(T_{0} \times v_{p}\right) \text {. }
$$

The prediction stage used here to maintain the ACC mode as long as possible to recover the track of the leader vehicle while the distance $d$ is above the vehicle length $L$. If the predicted distance is too short, then the STOP is called to exit the platooning mode. Otherwise, the vehicle can continue to rely on ACC mode.

While the preceding vehicle is correctly detected by the LRF system $(C \geq 90 \%)$, the updated VLC data are analyzed to evaluate whether the conditions are sufficient or not to activate a cooperative mode. The first criteria checks if all of the angles in eq. (4) satisfies the safety requirement of being less than the critical angle $\zeta \leq \theta_{c}$.

$$
\theta_{c}=0.8 \times \max (\varphi, \psi, \theta) .
$$

The second criteria checks in real time if the delay $\tau$ between any two consecutive received packets is below the maximum allowed time delay $(75 \mathrm{~ms})$. When these two criteria are satisfied, the algorithm outputs that it is safe to activate the CACC mode. However, when one of these conditions fails, only the ACC mode is allowed, if this is followed by any problems with the LRF requirements, the STOP state is called immediately to exit the platooning mode.

\section{Platforms Architecture}

Our system is evaluated using real VLC devices and a simulation environment for the LRF, vehicular controllers and car-following trajectories. Such experiment meant to validate the feasibility of our system for the platooning application before implementing the system in our experimental platforms.

\section{A. VLC Platform}

Based on our previous work [21] to realize the VLC dual functionality of both data transmission and vehicular lighting, 6 LEDs array with an emission wavelength of $635 \mathrm{~nm}$ were deployed to imitate vehicular rear-light as transmitter. As for the receiver end, the main components are the silicon Photo-Diode (PD) with a trans-impedance switchable gain PDA100A, combined with an optical band-pass filter and a direct detection decision circuit. Fig. 5 pictures the VLC principal components used in our study. 


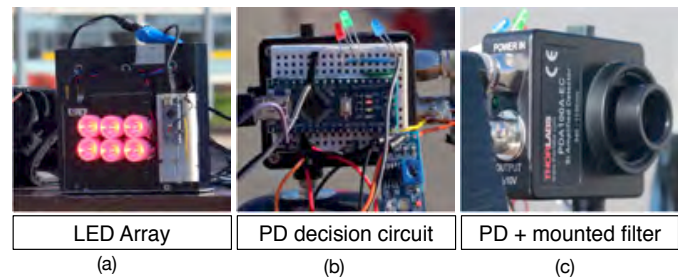

Fig. 5. VLC transmitter and receiver ends.

Moreover, a low-cost Mini-pro Arduino boards based on ATmega328P microcontroller has been used at both transmitter and receiver ends to perform modulation/demodulation, line coding and interfacing with vehicles controller. The exchanged vehicular status are packed into a particular packet format as shown in Fig. 6. These data are then adapt Manchester line coding in the micro-controller before modulating the LED array.

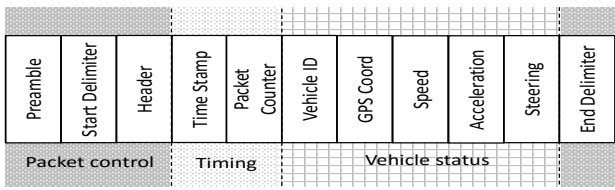

Fig. 6. VLC packet content.

\section{B. Pro-SiVIC Platform}

Our experiments intend to validate the uses cases and feasibility of the proposed approach for different road curvatures. This validation allow us to test the system over-all performance before the final implementation and integration into a real platforms. Hence, Pro-SiVIC, a simulation platform for vehicles, sensors, and environment is used to simulate two autonomous vehicles in three scenarios:

- Straight, the leader vehicle is driving in a straight line,

- Lane change, the leader vehicle is changing the lane at certain time instants.

- Curve, the leader vehicle reaches $90^{\circ}$ of orientation at crossroad during the simulation.

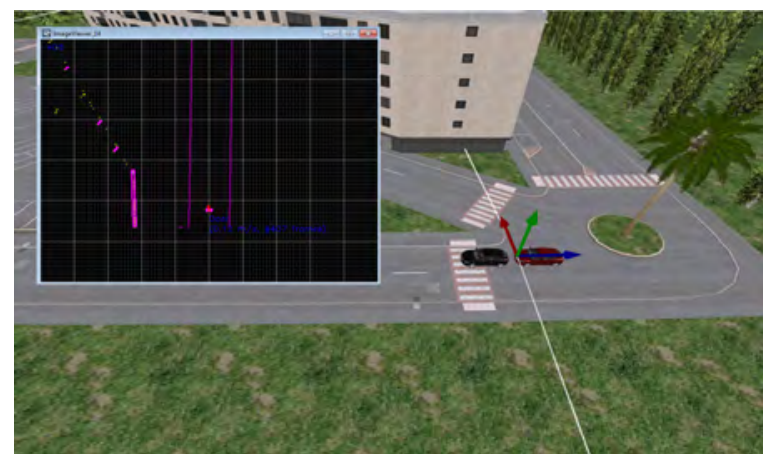

Fig. 7. Pro-SiVIC simulation platform.

Figure 7 illustrates the Pro-SiVIC simulation environment with the two vehicles, where the black one is the leader connected to the VLC transmitter, and the red one is the platoon member connected to the VLC receiver and equipped with a LRF to estimate the distance with the leader. The top left image in the same Figure, pictures sample of the follower vehicle detection using LRF. The simulation environment parameters of the Pro-SiVIC and the hardware details of the VLC platform are both listed in Table I

TABLE I

EXPERIMENTAL PARAMETERS

\begin{tabular}{ll}
\hline VLC Platform Parameter & Value \\
\hline Transmission data Rate & $9.5 \mathrm{kbps}$ \\
Link maximum range & $30 \mathrm{~meter}$ \\
LED wavelength & $635 \mathrm{~nm}$ (RED) \\
Line coding \& Modulation & Manchester, OOK \\
LED power dissipation & $3 \mathrm{Watt}$ \\
Number of LED array & 6 \\
LED lens viewing angle & $5^{\circ}, 15^{\circ}, 35^{\circ}, 45^{\circ}$ \\
PD active area & $100 \mathrm{~mm}^{2}$ \\
Transmitter \& Receiver heights & $85 \mathrm{~cm}$ \\
Gain levels & $30 \mathrm{~dB}, 40 \mathrm{~dB}$ \\
\hline Pro-SiVIC Parameters & Value \\
\hline Vehicle mass & $1500 \mathrm{~kg}$ \\
Vehicle length $(L)$ & $4.2 \mathrm{~m}$ \\
LRF range & $100 \mathrm{~m}$ \\
LRF angular aperture & $180 \circ$ \\
LRF angular resolution & $0.25 \circ$ \\
$W_{\text {Area }}$ & $9 \mathrm{~m}$ \\
\hline
\end{tabular}

\section{PERFormanCE EVALUATION}

The performance of the proposed platoon system deploying VLC and LRF is evaluated by driving platoon in the ProSiVIC simulator together with using an actual VLC prototype. The three main simulation scenarios represent different road curvature and vehicular maneuvers. The scenarios were meant to introduce communication and/or sensor failure and compare the platoon behavior with and without using VLC. For each of the listed scenarios, the VLC-FOV was set to $5^{\circ}, 15^{\circ}, 30^{\circ}$ and $45^{\circ}$, the LRF-FOV was set to $180^{\circ}$ with width of interest $W_{\text {Area }}=9 \mathrm{~m}$.

All the depicted results in Fig. (8, 9, 11) compare the leader vehicle speed (Leader Speed $_{\text {) }}$ with the received speed over VLC link at the follower side $\left(R X_{\text {Speed }}\right)$. Regardless the technology-in-use, either VLC or LRF, the results compare these speeds with the actual applied speed on the follower vehicle (Follower $\left._{\text {Speed }}\right)$, where the Speed $_{\text {difference }}$ for each VLC-FOV case is calculated by:

$$
\text { Speed }_{\text {difference }}=\text { Leader }_{\text {Speed }}-\text { Follower }_{\text {Speed }} .
$$

\section{A. Straight driving Scenario}

Figure 8 shows a sample of the simulation results for straight curvature scenario. The results manifest an agreement between Leader ${ }_{\text {Speed }}, R X_{\text {Speed }}$ and Follower $_{\text {Speed }}$, where none of the tracking angles $(\theta, \phi, \psi)$ exceeded the minimum $5^{\circ}$ limit, and the LRF confidence $C=100 \%$ allover the trajectory. Thus, follower vehicle always uses the

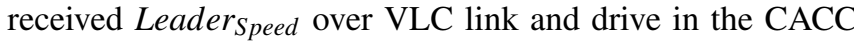
mode. 


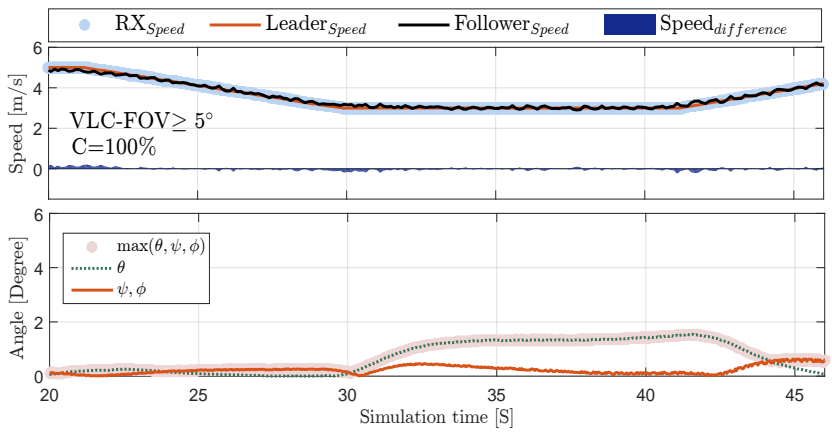

Fig. 8. Simulation results of Straight driving platoon scenario. laser based detection confidence $\mathrm{C}=100 \%$.

\section{B. Lane-Change Scenario}

The VLC link is more subjected to FOV misalignment when leader vehicle changes the lane. The results depicted in Fig. 9 show the smooth performance of the Follower $_{\text {Speed }}$ till the simulation time window 30-43s. The period when the leader vehicle began to change the lane until the follower vehicle reach aligned state. While the $\max (\theta, \psi, \phi)$ exceed the $\theta_{c}=0.80 * 5^{\circ}$, the disconnection prediction occur, and since $C=100 \%$, LRF will manage to backup and the driving mode switch from CACC to the ACC as described in the hand-over algorithm. For any VLC-FOV $\geq 30$, the system will behave as in the straight-lane scenario.

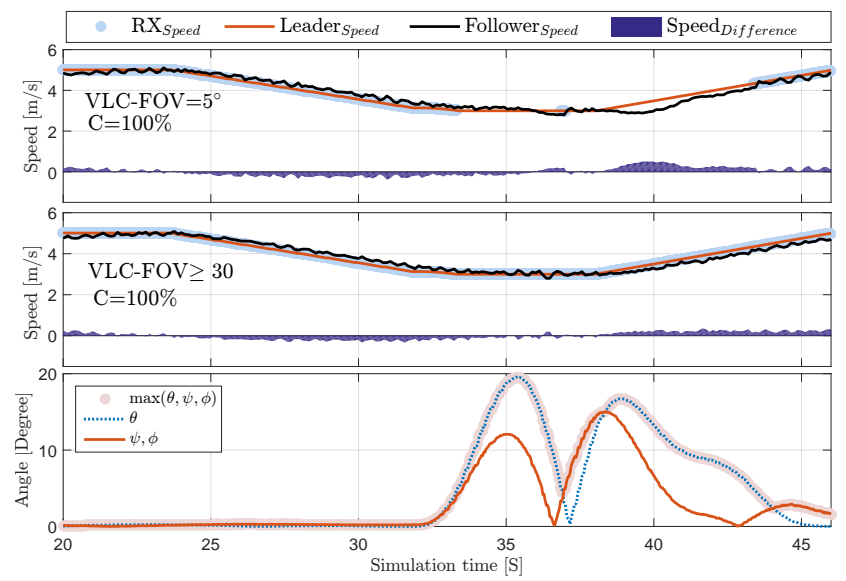

Fig. 9. Simulation results of Lane-change driving platoon scenario. laser based detection confidence $\mathrm{C}=100 \%$.

\section{Curve Scenario}

Compared to the other previous scenarios, the $90^{\circ}$ curve trajectory subjects VLC link to the maximum FOV misalignment and drives the follower vehicle close to the edge of LRF detection area. As depicted in Fig. 10, while the leader vehicle is turning at the boundary of the laser area of interest, the track continuity is not always maintained, and the LRF confidence parameter $\mathrm{C}$ will fluctuate between 0 and $100 \%$ for the first time compared with the previous other scenarios.

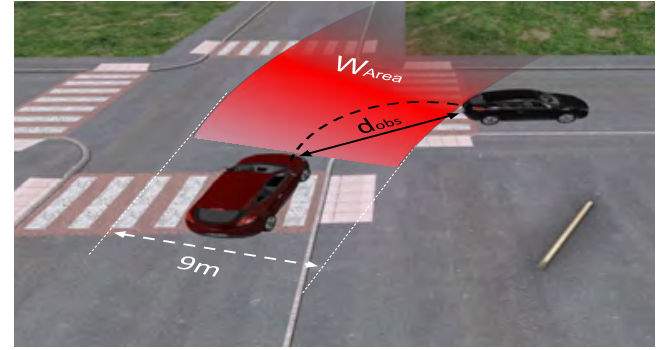

Fig. 10. Pro-SiVIC simulation case for $90^{\circ}$ curvature scenario, the platoon leader vehicle locate at the edge of the LRF of the follower vehicle.

Thus, the distance to the preceding vehicle predicted is based on the latest vehicle displacement. In this particular case, the Follower $_{\text {Speed }}$ slows down while the leader vehicle is not detected with $C \geq 90 \%$ over the time window $39.5-40.5 \mathrm{~s}$ for VLC-FOV $=5^{\circ}$ in Fig. 11. Better performance is observed for a VLC-FOV $\geq 45^{\circ}$, but the communication disconnection still occurs for shorter time period.

When $\mathrm{C}$ is falling below the $90 \%$ limit, a non-smooth behaviour of the Follower $_{\text {Speed }}$ is observed due to the fluctuations of the estimated inter-vehicle distance. Since VLC is not enabled here, the system will try to predict the distance and maintains the ACC mode as long as possible as described in the hand-over algorithm. After simulation time $40.5 \mathrm{~s}$ for $\mathrm{VLC}-\mathrm{FOV} \geq 45^{\circ}$, the leader vehicle is already detected with $\mathrm{C} \geq 90 \%$ and the VLC is enabled, which allows the system to activate the CACC mode again providing smoother Follower $_{\text {Speed }}$ response.

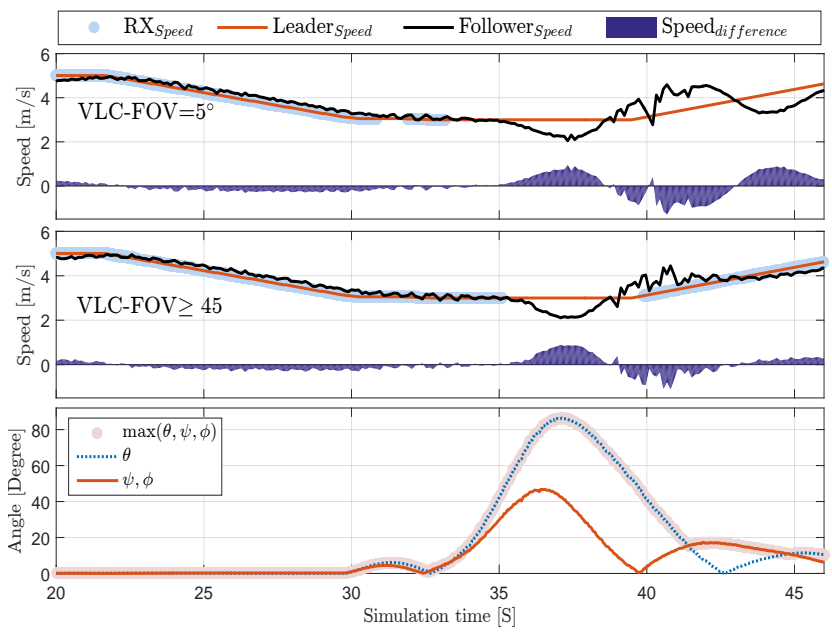

Fig. 11. Simulation results of Curve driving platoon scenario.

Figure 12 addresses the laser detection problem when the leader vehicle leaves the laser detection area of the following one. The results examine the narrowest VLC-FOV over the sharpest curvature $\left(90^{\circ}\right)$. Case 1 indicates the first occurrence of VLC disconnection due to the orientation limitation. During this period, the LRF is capable of substituting VLC and activate the ACC mode ensuring the safety requirements.

In case 2, the disconnection occurs again, but this time, the LRF will not be able to backup within a time delay satisfies 


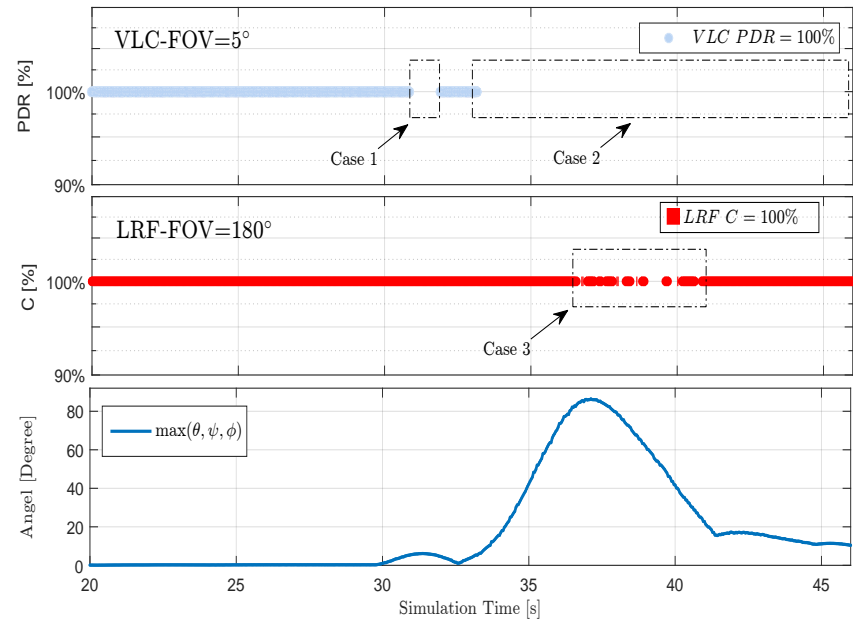

Fig. 12. PDR and C Simulation result of Curve driving platoon scenario.

the platoon safety requirements. The variation in $C$ as seen in case 3 occurs because the leader vehicle is repeatedly lost and recovered by the LRF of the follower and resulting in a non-smooth vehicle response. These results open the discussion to either use one more backup technology such as radio communication or go toward improving the laserbased solution.

\section{CONCLUSIONS}

In this work, we studied a combined solution using laserbased perception and visible light communication for platooning, where we proposed a hand-over algorithm profiting from both technologies. Although that LRF and VLC are independent of each other and provide a different type of information, the trajectory shape and vehicles orientation has a direct influence on both technologies. Indeed, our results show a perfect availability of cooperative platooning mode for low curve scenarios with $100 \%$ of PDR for VLC and $100 \%$ of confidence for LRC. However, these performances drop over extreme curves $\left(\geq 90^{\circ}\right)$ due to discontinuity in the VLC system, combined with fluctuations in LRF confidence value. Concerning LRF based perception, the main limitation comes from the difficulty to correctly identify the preceding vehicle. For such cases, using cameras to improve the vehicle recognition and tracking could tackle this limitation and improve the system performance.

Our future work focus on 1). Using the GPS data to improve the searching area of interest, 2). Efficient implementation of the hand-over algorithm by introducing radio communication to handle the system unmanaged failures by both technologies, 3 ). The tests of the overall system on real platforms, and finally 4). Establishing the proposed system performance using the state of the art of both ACC and CACC techniques in different conditions.

\section{ACKNOWLEDGMENT}

This study was supported in part by the research grant SINETIC of the French "Fonds Unique Interministriel" (FUI).

\section{REFERENCES}

[1] S. Tsugawa, "Inter-vehicle communications and their applications to intelligent vehicles: an overview," in Intelligent Vehicle Symposium, 2002. IEEE, vol. 2, June 2002, pp. 564-569 vol.2.

[2] S. H. Hosseinnia, I. Tejado, V. Milans, J. Villagr, and B. M. Vinagre, "Experimental application of hybrid fractional-order adaptive cruise control at low speed," IEEE Transactions on Control Systems Technology, vol. 22, no. 6, pp. 2329-2336, Nov 2014.

[3] D. Jiang and L. Delgrossi, "Ieee 802.11p: Towards an international standard for wireless access in vehicular environments," in Vehicular Technology Conference, 2008. VTC Spring 2008. IEEE, May 2008, pp. 2036-2040.

[4] Y. Wang, J. Hu, Y. Zhang, and C. Xu, "Reliability evaluation of ieee 802.11 -based vehicle-to-vehicle communication in an urban expressway," Tsinghua Science and Technology, vol. 20, no. 4, pp. 417-428, August 2015

[5] S. Shladover, "Path at 20 - history and major milestones," in Intelligent Transportation Systems Conference, 2006. ITSC 'O6. IEEE, 2006, pp. 122-129.

[6] P. Daviet and M. Parent, "Longitudinal and lateral servoing of vehicles in a platoon," in Intelligent Vehicles Symposium, 1996., Proceedings of the 1996 IEEE, 1996, pp. 41-46.

[7] S. Hall, B. Chaib-draa, and J. Laumonier, "Car platoons simulated as a multiagent system," in In: Proc. 4th Workshop on Agent-Based Simulation, 2003, pp. 57-63.

[8] E. C. Tom Robinson, Eric Chan, "Operating platoons on public motorways: An introduction to the sartre platooning programme," in 17th World Congress on Intelligent Transport Systems (ITS) 2010, October 2010.

[9] B. Schwarz, "LIDAR: Mapping the world in 3-D," Nature Photonics, vol. 4, no. 7, 2010 .

[10] B. Douillard, J. Underwood, N. Kuntz, V. Vlaskine, A. Quadros, P. Morton, and A. Frenkel, "On the segmentation of 3D LIDAR point clouds," in 2011 IEEE International Conference on Robotics and Automation, 2011.

[11] F. Nashashibi and A. Bargeton, "Laser-based vehicles tracking and classification using occlusion reasoning and confidence estimation," in IEEE Intelligent Vehicles Symposium, no. September, 2008.

[12] P. Fernandes and U. Nunes, "Platooning with dsrc-based ivc-enabled autonomous vehicles: Adding infrared communications for ivc reliability improvement," in Intelligent Vehicles Symposium (IV), 2012 IEEE, 2012, pp. 517-522.

[13] M. Y. Abualhoul, M. Marouf, O. Shag, and F. Nashashibi, "Enhancing the field of view limitation of visible light communication-based platoon," in Wireless Vehicular Communications (WiVeC), 2014 IEEE 6th International Symposium on, Sept 2014, pp. 1-5.

[14] M. Y. Abualhoul, M. Marouf, O. Shagdar, and F. Nashashibi, "Platooning control using visible light communications: A feasibility study," in 16th International IEEE Conference on Intelligent Transportation Systems (ITSC 2013), Oct 2013, pp. 1535-1540.

[15] A. Cailean, B. Cagneau, L. Chassagne, S. Topsu, Y. Alayli, and J.-M Blosseville, "Visible light communications: Application to cooperation between vehicles and road infrastructures," in 2012 IEEE Intelligent Vehicles Symposium (IV), 2012, pp. 1055-1059.

[16] S. Oncu, J. Ploeg, N. van de Wouw, and H. Nijmeijer, "Cooperative adaptive cruise control: Network-aware analysis of string stability," Intelligent Transportation Systems, IEEE Transactions on, vol. 15, no. 4, pp. 1527-1537, 2014.

[17] V. Milanés, S. E. Shladover, J. Spring, C. Nowakowski, H. Kawazoe, and M. Nakamura, "Cooperative adaptive cruise control in real traffic situations," Intelligent Transportation Systems, IEEE Transactions on, vol. 15 , no. 1, pp. 296-305, 2014.

[18] U. Department of Transportation, "Vehicle safety communications project task 3." http://www.ntis.gov/

[19] P. Merdrignac, O. Shagdar, I. Ben Jemaa, and F. Nashashibi, "Study on Perception and Communication Systems for Safety of Vulnerable Road Users," in 18th IEEE International Conference on Intelligent Transportation Systems (ITSC 2015), 2015.

[20] J. Kahn and J. Barry, "Wireless infrared communications," vol. 85, no. 2, pp. $265-298$, Feb. 1997.

[21] M. Abualhoul, O. Shagdar, and F. Nashashibi, "Visible Light Inter-Vehicle Communication for Platooning of Autonomous Vehicles," in 2016 IEEE Intelligent Vehicles Symposium IV2016, Gothenburg, Sweden, Jun. 2016. [Online]. Available: https://hal.inria.fr/hal-01308430 